\title{
Research
}

\section{Predicting clinical deterioration after initial assessment in out-of-hours primary care:}

\author{
a retrospective service evaluation
}

\begin{abstract}
Background

Accurate assessment of the need for admission is challenging in out-of-hours $(\mathrm{OOH})$ primary care. Understanding more about patient contacts where the decision to continue care in the community may have been incorrect could assist clinicians in assessing clinical risk.
\end{abstract}

Aim

To define the population contacting $\mathrm{OOH}$ primary care who are at higher risk of re-presenting to this service and requiring urgent transfer to secondary care within 3 days of their initial contact.

\section{Design and setting}

Retrospective service evaluation of 4 years of patient contacts with $\mathrm{Oxfordshire} \mathrm{OOH}$ primary care.

\section{Method}

Multivariable logistic regression was used to evaluate demographic and service delivery factors associated with increased risk of delayed escalation to secondary care.

\section{Results}

Almost 1\% of 496931 patients contacting $\mathrm{OOH}$ primary care required escalation to secondary care within 3 days. Of these, $68.5 \%$ were initially discharged with no follow-up or advice to contact their GP; $14.7 \%$ were initially referred to secondary care. The odds of requiring escalation were increased with age lodds ratio [OR] 1.010; 95\% confidence interval $[\mathrm{Cl}]=1.009$ to $1.011 ; P<0.001)$, more frequent prior use of the $00 \mathrm{H}$ service (OR $1.016 ; 95 \% \mathrm{Cl}=1.010$ to $1.021 ; P<0.001)$, and presenting during periods of low call volume IOR $0.880 ; 95 \% \mathrm{Cl}=0.857$ to 0.904; $P<0.001)$.

\section{Conclusion}

Older, prior users of the service, presenting at less busy times, are at greater risk of requiring secondary care referral from the $\mathrm{OOH}$ service within 3 days of their initial contact. These higher-risk patient groups might benefit from active follow-up by the $\mathrm{OOH}$ service.

\section{Keywords}

out-of-hours medical care; primary care; safety.

\section{INTRODUCTION}

The provision of primary care services outside core contracted hours is fundamental to the operation of the NHS. ${ }^{1}$ In 2013-2014, out-of-hours (OOH) GP services in England handled around 5.8 million cases, of which 3.3 million were face-toface consultations, including 800000 home visits. ${ }^{2}$ A challenge for $\mathrm{OOH}$ primary care is accurate assessment of clinical risk to guide a decision over whether to escalate a patient's care to hospital settings or to continue care in the community with advice on seeking further care (termed 'safety netting'). This assessment is more difficult in $\mathrm{OOH}$ primary care compared with in-hours routine general practice because of a lack of detailed medical records, lack of familiarity with the patient, increased use of telephone rather than face-to-face assessment, lack of support from trusted colleagues, and a higher proportion of vulnerable patients with more complex care needs. ${ }^{3}$

Furthermore, the decision to escalate care is complex. Decisions to admit patients from US emergency departments have been shown to be influenced by nonmedical factors in over half of cases. ${ }^{4}$ Age, sex, and ethnic group were associated with increased emergency admissions in a recent cross-sectional survey of admissions from in-hours general practice. ${ }^{5}$ In addition,

GN Hayward, MA, DPhil, MRCP, MRCGP academic clinical lecturer, Nuffield Department of Primary Care Health Sciences, University of Oxford; C Vincent, MPhil, PhD, patient safety lead, Oxford Academic Health Science Network, and professor of psychology, Department of Experimental Psychology, University of Oxford; DS Lasserson, MA, MD, FRCP Edin, MRCGP, senior interface physician in acute and complex medicine, NIHR Oxford Biomedical Research Centre, Oxford University Hospitals NHS Foundation Trust, John Radcliffe Hospital, and associate professor, Nuffield Department of Medicine, University of Oxford, Oxford. an individual GP's tolerance of risk affects the number of admissions made out-ofhours. ${ }^{6}$ These studies do not, however, offer insight into improving recognition of serious illness in $\mathrm{OOH}$ primary care. Mortality rates following $\mathrm{OOH}$ primary care assessment point to the higher prevalence of serious illness in this clinical population. In a region of Norway, $25 \%$ of patients had consulted local $\mathrm{OOH}$ primary care in the 4 weeks before their death, which was unexpected in a quarter of cases. ${ }^{7}$ There has been almost no research into other health outcomes following contact with $\mathrm{OOH}$ services.

Evidence that informs improvement in $\mathrm{OOH}$ care should consider recognition of patients who are at higher risk of clinical deterioration. Although safety-net advice can be given to all patients who are not escalated at initial assessment, identification of a patient group with a high likelihood of deterioration could drive alternative service models, including active review, rather than the default passive option of patientinitiated request for re-assessment. Highprofile failings in $\mathrm{OOH}$ care have resulted in part from a lack of active review models. ${ }^{8,9}$ Features identifying patients at higher risk of deterioration after initial assessment could be studied by examining those cases in which patients were kept at home following their initial assessment in $\mathrm{OOH}$ primary care and who re-attended within a short

\section{Address for correspondence}

Gail Hayward, Nuffield Department of Primary Care Health Sciences, Radcliffe Observatory Quarter, Woodstock Road, Oxford, OX2 6GG, UK. E-mail: gail.haywarddphc.ox.ac.uk

Submitted: 16 May 2016; Editor's response: 7 June 2016; final acceptance: 2 August 2016. CBritish Journal of General Practice

This is the full-length article (published online 8 Nov 2016) of an abridged version published in print. Cite this version as: Br J Gen Pract 2016; DOI: 10.3399/bjgp16X687961 


\section{How this fits in}

Out-of-hours $(\mathrm{OOH})$ primary care is a challenging clinical environment with acute illness syndromes. This study is the first to examine the 'failure rate' of $\mathrm{OOH}$ where patients return to the service and require escalation to secondary care within 3 days of initial contact. Older patients seen at times of low call volume have a higher risk of requiring escalation within 3 days. $\mathrm{OOH}$ providers should consider opportunities to develop proactive monitoring for patients at risk of deterioration after initial assessment.

time period, having clinically deteriorated. This study undertakes a service evaluation using data from the Oxfordshire $\mathrm{OOH}$ service to examine the population of patients who returned to the service within 3 days of an initial assessment and required hospitalbased care when re-assessed.

\section{METHOD}

The Oxfordshire out-of-hours electronic medical record was used to develop a database of patients presenting to the service over 4 years (June 2010 to August 2014). All patient identifiers were removed on entry to the database. Patients without an NHS number (14 572 patients) were not included in the database, as repeat visits to the service could not be assessed. The 75 patients who had presented more than 50 times to the service over a period of 4 years were censored at visit 50. Demographic data included age and Index of Multiple Deprivation (IMD) score. ${ }^{10}$ Service data included call volume, number of contacts before the index consultation, and time period.

Contact volume was assessed using the total number of contacts received for each hour of the day over the 4-year period. Contact volume was categorised as follows:
Figure 1. Outcomes of patient contacts with $\mathrm{OOH}$ primary care and the proportions of patient contacts with each outcome where escalation of care was required within 3 days. $\mathrm{OOH}=$ out-of-hours. low <2500, moderate 2500-5000, high $\geq 5000-10000$, and very high $\geq 10000$. The number of days' difference was calculated using calendar days beginning at midnight. Patients who had two separate contacts with the service within the same 1-hour time period were excluded from the analysis. Where more than two contacts were made with the service within 3 days, the earliest contact was taken as the initial contact.

Demographic and service characteristics were determined that were independently associated with referral to secondary providers (hospital admission, emergency department referral, acute ophthalmology, or psychiatry referral, or 999 ambulance) at re-attendance to the $\mathrm{OOH}$ service within 3 days of their initial visit with multivariable logistic regression. Data on referral to secondary care providers from the in-hours GP or ambulance, or direct presentations to secondary care services, were not available to this analysis. Analyses were performed using SPSS (version 22).

\section{Validation of clinical coding}

At the end of each $\mathrm{OOH}$ consultation, clinicians assign at least one clinical code (for example 'cardiac'), which were used in this study to determine clinical presentation for that contact. To validate the clinical codes applied by the $\mathrm{OOH}$ clinicians, estimates were made, based on previous coding validity studies, ${ }^{11}$ that 230 records would be required to establish the coding validity with a confidence level of $90 \%$ and $5 \%$ margin of error. A random selection of 230 records was obtained using SPSS, and the clinical code was compared by one author to the conclusion drawn by the clinician in the medical notes. The positive predictive value (PPV) of the clinical code for medical diagnosis or conclusion was $90 \%$. If only those codes that positively described an established clinical presentation were assessed (for example, 'cardiac' but not 'miscellaneous' or 'referred to acute trust'), the PPV was $97.5 \%$.

\section{RESULTS}

Of 496931 presentations to the $\mathrm{OOH}$ service, $58.3 \%$ were female, with median age 35 years linterquartile range [IQR] 13-66 years). Of this population, $78 \%$ were discharged with no follow-up or advised to contact their own GP. Almost 1\% (4832 cases, 4465 individuals) of this population (57.5\% female) required escalation to secondary care providers within 3 days of their initial visit: the 'delayed escalation' population. The flow of the patients through the service is described in Figure 1. The 
Table 1. Demographics of the overall population and 'delayed escalation' population

\begin{tabular}{lcc} 
& $\begin{array}{c}\text { Overall patient } \\
\text { contacts }\end{array}$ & $\begin{array}{c}\text { 'Delayed escalation' } \\
\text { patient contacts }\end{array}$ \\
\hline Sex & $58.3 \%$ female & $57.5 \%$ female \\
\hline Age, years, median $[\mathrm{IQR}]$ & $35[13-66]$ & $52[22-78]$ \\
\hline IMD, mean [range], SD & $14.1(0.6-84.2), 10.3$ & $13.9(1.1-70.4], 10.1$ \\
\hline Number of OOH contacts before & $1[0-2]$ & $1[1-3]$ \\
the index consultation, median [IQR] & & \\
\hline IMD = Index of Multiple Deprivation. IQR = interquartile range. OOH = out-of-hours. SD = standard deviation.
\end{tabular}

Table 2. Factors included in logistic regression analysis

\begin{tabular}{lccc} 
& OR $(\mathbf{9 5 \%} \mathbf{C l})$ & Wald & $\boldsymbol{P}$-value \\
\hline Age & $1.010(1.009$ to 1.011$)$ & 417.3 & 0.000 \\
\hline Sex & $1.05(0.991$ to 1.113$)$ & 2.7 & 0.101 \\
\hline Deprivation & $1.000(0.997$ to 1.003$)$ & 0 & 0.982 \\
\hline OOH contacts before index consultation & $1.016(1.010$ to 1.021$)$ & 33.5 & 0.000 \\
\hline Call volume & $0.880(0.857$ to 0.904$)$ & 89.4 & 0.000 \\
\hline OOH = out-of-hours. OR $=$ odds ratio. & & &
\end{tabular}

Table 3. Clinical presentations associated with highest risk of requiring 'delayed escalation"

\begin{tabular}{lccc} 
& $\begin{array}{c}\text { Consultations with } \\
\text { clinical presentation } \\
\text { in 'delayed } \\
\text { escalation' group (\%) }\end{array}$ & $\begin{array}{c}\text { Total number of } \\
\text { consultations } \\
\text { with clinical } \\
\text { presentation (\%) }\end{array}$ & $\begin{array}{c}\text { \% risk of 'delayed } \\
\text { escalation'a }\end{array}$ \\
\hline Social & $35(0.7)$ & $1631(0.3)$ & 2.15 \\
\hline Mental health & $231(4.8)$ & $11003(2.2)$ & 2.10 \\
\hline Abnormal pathology result & $17(0.4)$ & $950(0.2)$ & 1.79 \\
\hline Cardiac & $129(2.7)$ & $7470(1.5)$ & 1.73 \\
\hline Gastrointestinal & $845(17.5)$ & $51788(10.4)$ & 1.63 \\
\hline${ }^{a}$ Number of patient contacts with this clinical presentation who were escalated within 3 days/total patient \\
contacts with this clinical presentation $\times 100$.
\end{tabular}

demographics of the overall and delayed escalation' populations are described in Table 1.

The 'delayed escalation' population made initial contact with the service most often on Saturdays (1837 out of 4832 contacts; 38\%), although the risk of delayed escalation was greatest on Fridays $11.4 \%$ of total Friday contacts). Almost half (48.4\%) of urgent referrals to secondary care at re-attendance were acute hospital admissions made directly to a specialty. Emergency department referrals accounted for $29.7 \%$, 999 ambulances $18.3 \%$, acute psychiatry $2.8 \%$, and ophthalmology $0.8 \%$.
Factors increasing risk of referral to secondary care within 3 days

Multivariable logistic regression was used to test which demographic and service delivery factors were associated with initial patient contacts that resulted in delayed escalation to secondary care within 3 days. All of the demographic factors available from the database were included, and service variables that uniquely identified features of service demographics. As contact volumes were universally lower in the overnight period, contact volume was included in the analysis without an additional variable for time period.

Significant factors were increasing age, higher frequency of attendance to the $\mathrm{OOH}$ service before this contact, and when presenting to the service during periods of low contact volumes, mostly in the overnight period (Table 2). The same factors were significant when the analysis was restricted to those patients who were not referred to secondary care following their initial contact (4122 patient contacts) (Appendix 1).

\section{Risk of delayed escalation by clinical problem}

Patients presenting with social, mental health, gastrointestinal, and cardiac problems carried the highest risk of re-presentation with deterioration, in addition to those contacted because of abnormal pathology results (Table 3 ).

\section{Clinical problems at the initial consultation and at re-attendance in the delayed} escalation population

Table 4 displays the top 10 most common clinical presentations for the delayed escalation' patient group at their initial consultation and at re-attendance. The highest-frequency presentations were gastrointestinal, genitourinary, respiratory, and musculoskeletal at both the initial consultation and on re-attendance. More patients presented with surgical, cardiac, neurological, and respiratory problems at the contact that resulted in escalation of care.

\section{Risk of delayed escalation by consultation} outcomes

Referral to acute psychiatric services was the outcome of contact with $\mathrm{OOH}$ that carried the greatest risk of re-assessment with hospital admission within 3 days. This suggests that patients were accepted for further assessment by the psychiatry services but were discharged back to community care within 3 days.

Other consultation outcomes associated with higher risk were referral to social 
Table 4. The 10 most commonly assigned clinical codes on initial consultation and re-attendance

\begin{tabular}{|c|c|c|c|c|}
\hline \multirow[b]{2}{*}{ Rank } & \multicolumn{2}{|c|}{ Initial consultation } & \multicolumn{2}{|c|}{ Re-attendance } \\
\hline & $\begin{array}{c}\text { Clinical } \\
\text { presentation }\end{array}$ & $\begin{array}{c}\text { Number of } \\
\text { consultations }(\%)\end{array}$ & $\begin{array}{c}\text { Clinical } \\
\text { presentation }\end{array}$ & $\begin{array}{c}\text { Number of } \\
\text { consultations (\%) }\end{array}$ \\
\hline 1 & Gastrointestinal & 845 (15.8) & Gastrointestinal & $813(15.0)$ \\
\hline 2 & Genitourinary & $565(10.6)$ & Genitourinary & $513(9.5)$ \\
\hline 3 & Respiratory & $320(6.0)$ & Respiratory & $511(9.4)$ \\
\hline 4 & Musculoskeletal & $303(5.7)$ & Musculoskeletal & 299 (5.5) \\
\hline 6 & Ophthalmic or ENT & 210 (3.9) & Surgical & $249(4.6)$ \\
\hline 7 & Viral infection or flu-like illness & 205 (3.8) & Cardiac & 233 (4.3) \\
\hline 8 & Local infection & 197 (3.7) & Mental health & $231(4.3)$ \\
\hline 9 & Lower respiratory tract infection & 179 (3.3) & Ophthalmic or ENT & $212(3.9)$ \\
\hline 10 & Obstetrics and gynaecology & $142(2.7)$ & Local infection & 207 (3.8) \\
\hline
\end{tabular}

services, 999 ambulance referrals, consultations where the patient left after triage but before definitive assessment, and patients where the $\mathrm{OOH}$ service had planned to follow-up their care (Table 5).

\section{Outcomes from the initial consultation}

A third (32.2\%) of 'delayed escalation' patient contacts were advised to see their own GP for review, and over a third (36.3\%) were discharged with no follow-up planned. The proportions of patients given this advice in the overall population contacting $\mathrm{OOH}$ were $31.5 \%$ and $46.6 \%$, respectively. Patients in the 'delayed escalation' group were referred to secondary care at their initial consultation almost twice as often as the overall population $(14.7 \%$ versus $8.3 \%)$; these patients were discharged back to the community only to require escalation back to hospital within 3 days. In the group of patients referred at their initial contact, $42.5 \%$ were referred to

\section{Table 5. Consultation outcomes associated with the highest risk of} requiring 'delayed escalation'

\begin{tabular}{|c|c|c|c|}
\hline $\begin{array}{l}\text { Consultation } \\
\text { outcome }\end{array}$ & $\begin{array}{l}\text { Consultations with } \\
\text { this outcome } \\
\text { in 'delayed } \\
\text { escalation' group }\end{array}$ & $\begin{array}{l}\text { Consultations with } \\
\text { this outcome } \\
\text { in patients } \\
\text { not escalated }\end{array}$ & $\begin{array}{l}\% \text { risk of } \\
\text { 'delayed } \\
\text { escalation'a }\end{array}$ \\
\hline Acute psychiatry & $33(0.68 \%)$ & 808 (0.17\%) & 3.92 \\
\hline 999 ambulance & 302 (6.25\%) & 9203 (1.91\%) & 3.50 \\
\hline Follow-up planned by $\mathrm{OOH}$ service & 169 (3.48\%) & 7717 (1.59\%) & 2.13 \\
\hline Social services & $2(0.04 \%)$ & $114(0.02 \%)$ & 1.72 \\
\hline Triaged to base visit but left before treatment & $7(0.14 \%)$ & $425(0.09 \%)$ & 1.62 \\
\hline
\end{tabular}

hospital by emergency ambulance, 24.1\% were acute hospital admissions made directly to a specialty, $27.5 \%$ were referred to the emergency department, and $4.6 \%$ and $1.3 \%$ were referred to acute psychiatry and acute ophthalmology, respectively.

Contacts were mainly during daytime weekend shifts (60.8\%). Of the patients, $55.6 \%$ were female and the median age was 57 years (IQR 24-78 years).

\section{DISCUSSION}

\section{Summary}

Almost one per cent of patients required urgent admission to secondary care within 3 days of an initial consultation in this UK $\mathrm{OOH}$ primary care service. Patients who were older, had used the service more often, and presented to the service at times of low calls were at higher risk. Over two-thirds of the patients who required escalation of care after re-attending within 3 days were initially discharged with either no follow-up or advice to contact their own GP.

Patients presenting with social, mental health, gastrointestinal, and cardiac problems were at highest risk of requiring delayed escalation of care. The social descriptor is often used to indicate a functional decline, which means that the patient can no longer manage in their usual home environment without a clear medical cause. This may indicate that acute illness can initially present as a functional decline, particularly for frail, older patients with multimorbidity, and a medical diagnosis can become more manifest over ensuing days.

\section{Strengths and limitations}

This is the first assessment of factors 
associated with risk of clinical deterioration in $\mathrm{UK} \mathrm{OOH}$ care. The evaluation included a large volume of patient contacts with validated clinical coding. An important limitation is that full data linkage with in-hours GP notes and Accident and Emergency or other urgent care contacts is not systematically available in the UK, therefore the present data are likely to significantly underestimate the number of patients who will be referred urgently to secondary care within 3 days, because many more will be referred by their usual GP. The percentage risk of delayed escalation was greatest for Friday contacts (1.4\%), when patients are reliant on $\mathrm{OOH}$ primary care in event of deterioration within 3 days. This is likely to better approximate the true rate of re-presentation.

\section{Comparison with existing literature}

The present findings mirror research based in emergency department settings, where older adults use emergency services at a higher rate and are more likely to be admitted or to re-attend than younger adults. ${ }^{12}$ In an emergency department short-stay unit, age but not overnight presentation was associated with rapid re-presentation to acute care following discharge. The present finding that 'gastrointestinal' was the most common clinical presentation in this patient group matches prehospital triage research showing that 'acute abdominal' cases had the highest chance of being initially 'undertriaged' by telephone, and then upgraded to more severe on face-toface assessment. ${ }^{13}$

\section{Implications for research and practice}

The present analysis suggests a number of measures that could reduce the number of patients who have delayed escalation to secondary care, which could be applied either separately or in combination.

Presentation at times of low call volume for the $\mathrm{OOH}$ service was associated with higher risk of 'delayed escalation'. Periods of low call volume are commonly covered by smaller numbers of clinicians. Therefore, the pressures of the service may lead to deferred decision-making by telephone triage until more staff are available, for example, to offer a home visit the following day. Norwegian $\mathrm{OOH}$ GPs described the challenges of balancing practical issues and clinical need when offering home visits with limited clinician resources. ${ }^{14}$ The GP's tolerance of risk has been shown to be an important factor for decisions about admission from $\mathrm{OOH}$ care. GPs selfrating as cautious refer more often than those rating themselves as good at living with risk and uncertainty. ${ }^{6}$ GPs choosing to work in overnight $\mathrm{OOH}$ shifts, which are considered to be more pressured, may be a selected group of clinicians who have higher risk tolerance. Increasing the number of clinicians available in this time period may allow more timely review of higher-risk patient groups and encourage more clinicians to consider these shifts.

Over two-thirds of the "delayed escalation population were discharged at their initial consultation and just 3.5\% were offered follow-up by the $\mathrm{OOH}$ service. Active followup could allow $\mathrm{OOH}$ services to detect deterioration earlier, and offer the potential for enhanced input to meet clinical need without an emergency admission. The patient group targeted for follow-up should include older patients, particularly those presenting with unexplained functional decline, patients initially presenting overnight, and patients with mental health, cardiac, and gastrointestinal problems where there is diagnostic doubt. A records-based follow-up list, with a planned telephone review period of 12 or 24 hours, could be employed. This would be particularly beneficial over the weekend, when the $\mathrm{OOH}$ service has continuity of care, and could also allow secondary care to highlight patients requiring community follow-up after discharge.

Patients who had contacted the $\mathrm{OOH}$ service previously were more likely to require escalation of care within 3 days. In European $\mathrm{OOH}$ services these patients have been shown to have higher occurrences of chronic disease and psychiatric illness; ${ }^{15,16}$ these are populations in which the decision to admit is likely to be more challenging. Enhanced sharing of information with the in-hours GP practice could facilitate shared management plans and reduce the risk of deterioration out-of-hours. Similarly, the present finding that $14.7 \%$ of the delayed escalation' population had been referred to secondary care at their initial consultation suggests that feedback on the outcomes of admissions from the $\mathrm{OOH}$ service would be highly beneficial in informing further management of these challenging patients. At present, $\mathrm{OOH}$ clinicians have no access to feedback on the decisions they make regarding referral. They would not learn about subsequent referral to secondary care, an immediate discharge following an admission, or even, unless it was particularly noteworthy, a death. Without such feedback, clinicians are unable to learn from their experiences in this higher risk setting. 


\section{Funding}

Gail N Hayward was funded by an NIHR Academic Clinical fellowship and Academic Clinical Lectureship while working on this study. Daniel $\mathrm{S}$ Lasserson is funded by the NIHR Oxford Biomedical Research Centre and NIHR Oxford Collaboration for Leadership in Applied Health Research and Care (CLAHRC). Charles Vincent is funded by the Health Foundation. No additional funding was sought for this study. The authors' funders had no role in study design; in the collection, analysis, and interpretation of data; in the writing of the report; or in the decision to submit the article for publication. The researchers are entirely independent from the funders.

\section{Ethical approval}

Ethical approval was not required for this study. Oxford Health NHS Foundation Trust evaluated the protocol and prospectively approved the work as a service evaluation and quality improvement project, conducted by Gail $N$ Hayward who was part of the direct care team.

\section{Provenance}

Freely submitted; externally peer reviewed.

\section{Competing interests}

The authors have declared no competing interests.

\section{Acknowledgements}

The authors acknowledge the input and assistance of Ms Helen Hunt, Urgent and Ambulatory Care Service Clinical Lead for Oxford Health NHS Foundation Trust, in this project.

\section{Discuss this article}

Contribute and read comments about this article: bjgp.org/letters
Improving the detection and management of patients who are on a declining trajectory requires research to understand the presenting features, the final diagnosis, and the outcome of escalation of care in this patient group. Improving care delivery through support for decision-making and follow-up models requires research into the type of clinicians used in $\mathrm{OOH}$ care pathways, availability of diagnostic testing, staffing levels at the time of assessment, and optimal timing of clinical review. This will allow $\mathrm{OOH}$ follow-up to be targeted at the highest-risk patients, and will enable development of decision support such as use of point-of-care testing, which could identify conditions notoriously difficult to detect clinically at an early stage (for example, acute kidney injury), as well as reduce inappropriate referral decisions. This will allow understanding of the impact of clinician experience and pressure on referral decisions and evaluation of whether the admissions were clinically justified or could have been prevented with alternative management strategies.

The present findings need to be confirmed and extended through multicentre studies or collaborations between different $\mathrm{OOH}$ service providers covering different UK populations that share data on re-attendance and escalation. Furthermore, definitive clinical outcome data after escalation to secondary care will be most efficiently gathered through local data-sharing partnerships between an $\mathrm{OOH}$ provider and secondary care organisations to enable linkage of patient data. New models of care incorporating active review by the $\mathrm{OOH}$ service will also need to be evaluated alongside routine care from different $\mathrm{OOH}$ service providers to assess their clinicaland cost-effectiveness. It is appreciated that increasing the number of GPs available in $\mathrm{OOHs}$ services, however desirable, is not easy at the present time. Understanding how to support decision-making by different healthcare professionals assessing patients in an $\mathrm{OOH}$ service, focusing more directly on those patients who are particularly at risk of missing a needed referral, and putting in place more active follow-up when such patients are seen, are potential strategies, however, to improve the safety of $\mathrm{OOH}$ primary care. 


\section{REFERENCES}

1. Baker M, Thomas M, Mawby R. The future of GP out of hours care. 2014. http:// wuw.rcgp.org.uk/policy/ /media/Files/Policy/A-Z-policy/RCGP-The-Future-ofGP-Out-of-Hours-Care-2015.ashx (accessed 24 Oct 2016).

2. National Audit Office. Out-of-hours GP services in England. London: National Audit Office, 2014. http://www.nao.org.uk/wp-content/uploads/2014/09/Out-ofhours-GP-services-in-England1.pdf laccessed 24 Oct 2016).

3. Colin-Thomé D, Field S. General practice out-of-hours services: project to consider and assess current arrangements. 2010. http://webarchive. nationalarchives.gov.uk/20130107105354/http://www.dh.gov.uk/prod_consum dh/groups/dh_digitalassets/dadh/den/_aps/documents/digitalasset/dh_111893. pdf (accessed 24 Oct 2016).

4. Lewis Hunter AE, Spatz ES, Bernstein SL, Rosenthal MS. Factors influencing hospital admission of non-critically ill patients presenting to the emergency department: a cross-sectional study. J Gen Intern Med 2016; 31(1): 37-44.

5. Gunther S, Taub N, Rogers S, Baker R. What aspects of primary care predict emergency admission rates? A cross sectional study. BMC Health Serv Res 2013; 13: 11.

6. Ingram JC, Calnan MW, Greenwood RJ, et al. Risk taking in general practice: GP out-of-hours referrals to hospital. Br J Gen Pract 2009; DOI: 10.3399/ bjgp09X394824.

7. Kristoffersen JE. Out-of-hours primary care and the patients who die. A survey of deaths after contact with a suburban primary care out-of-hours service. Scand J Prim Health Care 2000; 18(3): 139-142.

8. Department of Health. Response to the report of the panel overseeing the serious untoward incident investigation into the death of Penny Campbell. 4
September 2007. http://webarchive.nationalarchives.gov.uk/20130107105354/ http://www.dh.gov.uk/en/Publicationsandstatistics/Lettersandcirculars/ Dearcolleagueletters/DH_078343 (accessed 24 Oct 2016).

9. Mum dies three days after flu diagnosis. Ipswich Star 2009; 8 Jan: http://wnw. ipswichstar.co.uk/news/mum_dies_three_days_after_flu_diagnosis_1_169525 laccessed 24 Oct 2016).

10. Department for Communities and Local Government. English Indices of Deprivation 2010. London: Department for Communities and Local Government, 2011. https://www.gov.uk/government/statistics/english-indicesof-deprivation-2010 laccessed 24 Oct 2016).

11. Khan NF, Harrison SE, Rose PW. Validity of diagnostic coding within the General Practice Research Database: a systematic review. Br J Gen Pract 2010; DOI: 10.3399/bjgp10X483562.

12. Aminzadeh F, Dalziel WB. Older adults in the emergency department: a systematic review of patterns of use, adverse outcomes, and effectiveness of interventions. Ann Emerg Med 2002; 39(3): 238-247.

13. Rørtveit S, Meland E, Hunskaar S. Changes of triage by GPs during the course of prehospital emergency situations in a Norwegian rural community. Scand J Trauma Resusc Emerg Med 2013; 21: 89.

14. Brennvall HM, Hauken H, Hunskår S, et al. Out-of-hours doctors' decisions on call-outs in emergency situations. Tidsskr Nor Laegeforen 2015; 135(7): 654-657.

15. den Boer-Wolters D, Knol MJ, Smulders K de Wit NJ. Frequent attendance of primary care out-of-hours services in the Netherlands: characteristics of patients and presented morbidity. Fam Pract 2010; 27(2): 129-134.

16. Christensen MB, Christensen B, Mortensen JT, Olesen F. Intervention among frequent attenders of the out-of-hours service: a stratified cluster randomized controlled trial. Scand J Prim Health Care 2004; 22(3): 180-186. 
Appendix 1. Clinical presentations associated with highest risk of requiring 'delayed escalation' in the subgroup of patients where care was not escalated on their initial contact

\begin{tabular}{lccc} 
& OR $(\mathbf{9 5} \%$ CI) & Wald & $\boldsymbol{P}$-value \\
\hline Age & $1.010(1.009$ to 1.011$)$ & 323.16 & 0.000 \\
\hline Sex & $1.035(0.972$ to 1.103$)$ & 1.16 & 0.281 \\
\hline Deprivation & $1.000(0.997$ to 1.003$)$ & 0.02 & 0.886 \\
\hline OOH contacts before index consultation & $1.018(1.012$ to 1.024$)$ & 38.8 & 0.000 \\
\hline Call volume & $0.902(0.877$ to 0.929$)$ & 49.0 & 0.000 \\
\hline OOH = out-of-hours. OR =odds ratio. & & &
\end{tabular}

